\title{
Evidence Against Dark Matter Halos Surrounding the Globular Clusters Mgc1 and Ngc 2419
}

\section{Citation}

Conroy, Charlie, Abraham Loeb, and David N. Spergel. 2011. "EVIDENCE AGAINST DARK MATTER HALOS SURROUNDING THE GLOBULAR CLUSTERS MGC1 AND NGC 2419." The Astrophysical Journal 741 (2): 72. https://doi.org/10.1088/0004-637x/741/2/72.

\section{Permanent link}

http://nrs.harvard.edu/urn-3:HUL.InstRepos:41393159

\section{Terms of Use}

This article was downloaded from Harvard University's DASH repository, and is made available under the terms and conditions applicable to Other Posted Material, as set forth at http:// nrs.harvard.edu/urn-3:HUL.InstRepos:dash.current.terms-of-use\#LAA

\section{Share Your Story}

The Harvard community has made this article openly available.

Please share how this access benefits you. Submit a story.

Accessibility 


\title{
EVIDENCE AGAINST DARK MATTER HALOS SURROUNDING THE GLOBULAR CLUSTERS MGC1 AND NGC 2419
}

\author{
Charlie Conroy ${ }^{1}$, Abraham Loeb $^{1}$, and David N. Spergel ${ }^{2}$ \\ ${ }^{1}$ Harvard-Smithsonian Center for Astrophysics, Cambridge, MA, USA \\ ${ }^{2}$ Department of Astrophysical Sciences, Princeton University, Princeton, NJ, USA \\ Received 2011 August 3; accepted 2011 August 3; published 2011 October 18
}

\begin{abstract}
The conjecture that the ancient globular clusters (GCs) formed at the center of their own dark matter (DM) halos was first proposed by Peebles in 1984 and has recently been revived to explain the puzzling abundance patterns observed within many GCs. In this paper, we demonstrate that the outer stellar density profile of isolated GCs is very sensitive to the presence of an extended dark halo. The GCs NGC 2419, located at $90 \mathrm{kpc}$ from the center of our Galaxy, and MGC1, located at $\sim 200 \mathrm{kpc}$ from the center of M31, are ideal laboratories for testing the scenario that GCs formed at the centers of massive dark halos. Comparing analytic models to observations of these GCs, we conclude that these GCs cannot be embedded within dark halos with a virial mass greater than $10^{6} M_{\odot}$, or, equivalently, the DM halo-mass-to-stellar mass ratio must be $M_{\mathrm{DM}} / M_{*}<1$. If these GCs have indeed orbited within weak tidal fields throughout their lifetimes, then these limits imply that these GCs did not form within their own dark halos. Recent observations of an extended stellar halo in the GC NGC 1851 are also interpreted in the context of our analytic models. Implications of these results for the formation of GCs are briefly discussed.
\end{abstract}

Key words: dark matter - globular clusters: general

Online-only material: color figures

\section{INTRODUCTION}

Despite decades of intense theoretical effort, the formation of the ancient globular clusters (GCs) remains a largely unsolved problem. Peebles (1984) considered the possibility that GCs form within their own dark matter (DM) halos at high redshift. The growing evidence for significant self-enrichment in GCs and the broad acceptance of hierarchical structure formation has deepened interest in this formation scenario. Evidence against this scenario was found in the observations of thin tidal tails surrounding many GCs (e.g., Grillmair et al. 1995; Odenkirchen et al. 2003), because numerical simulations showed that such tidal tails do not form if GCs reside within extended halos (Moore 1996). However, later work highlighted the fact that even if Milky Way (MW) GCs were once embedded within massive dark halos, these halos would have been tidally stripped away by the present epoch (Bromm \& Clarke 2002; Mashchenko \& Sills 2005). This requires relatively strong tidal fields, which suggests that GCs in the outer halo of the MW may still be embedded within dark halos, if they formed within them.

Other theories for the formation of GCs do not appeal to formation at the center of dark halos. Fall \& Rees (1985) proposed that GCs form from thermal instabilities in the hot gaseous halos expected to surround massive galaxies today. This proposal suffers from the fact that many galaxies that host GCs are not expected to reside in halos massive enough to support a hot halo, such as dwarf spheroidals.

Gunn (1980) was the first to suggest that GCs could form in the gas compressed by strong shocks. This proposal received tentative confirmation with the discovery of many massive young star clusters within the interacting Antennae system (Whitmore \& Schweizer 1995; Whitmore et al. 1999) and the discovery of super star clusters within nearby galaxies (e.g., Holtzman et al. 1992). This scenario, modified to include as formation sites any massive, dense, cold patch of gas, is now the prevailing paradigm for GC formation (e.g., Harris \& Pudritz 1994), and, when incorporated into our broader theory of cosmological structure formation, is capable of explaining a variety of observations (e.g., Ashman \& Zepf 1992; Kravtsov \& Gnedin 2005; Muratov \& Gnedin 2010).

This prevailing paradigm for GC formation is complicated by the existence of nuclear star clusters (Böker et al. 2004; Walcher et al. 2005, 2006), which implies that at least some GC-like systems can form at the centers of massive dark halos. The existence of young nuclear star clusters makes this point particularly compelling, since these clusters could not have migrated to the center via dynamical friction. Thus, while dark halos are not necessarily required for GC formation, the conditions for GC formation may sometimes be realized at the centers of dark halos. Clearly, further constraints on the formation sites of GCs is desirable.

In a series of papers, Spitzer and collaborators derived the kinematic properties of stars in the stellar halo of a GC, where stars are only marginally bound (Spitzer \& Hart 1971; Spitzer \& Shapiro 1972). An important result from this work was that the density profile of stars in the stellar halo should scale as $r^{-3.5}$. In the present work, we build upon these results by investigating the sensitivity of the stellar density profile to the presence of a massive dark halo.

\section{THE STELLAR HALOS OF GLOBULAR CLUSTERS}

\subsection{Analytic Model}

In this section, we derive the outer stellar density profile of GCs embedded in a massive dark halo. The following derivation closely follows the assumptions and approximations made in a series of papers by Spitzer and collaborators (Spitzer \& Hart 1971; Spitzer \& Shapiro 1972; Spitzer 1987), to which the reader is referred for details. 
The density profile of a stellar system can be derived from its distribution function, $f$, via

$$
n(r) \propto \int_{E<0} f(E, J) 2 \pi v_{t} d v_{t} d v_{r},
$$

where $v_{t}$ and $v_{r}$ are the tangential and radial velocities. We assume that GC halo stars are on radial orbits, and thus are justified in making the approximation that $v^{2}=v_{r}^{2}$, and we can substitute $v_{t}=J / r$. Most importantly, we assume that $f(E, J)=|E| g(J)$, where $g$ is some function of angular momentum. This functional form arises when the orbital energies are only slightly below zero, the number of stars in the system is large, and the system has reached a steady state (see Spitzer $\&$ Shapiro 1972, for details). These constraints require that the two-body relaxation time is short compared to the age of the universe. We then have

$$
n(r) \propto r^{-2} g^{\prime}(J) \int_{E<0}|E| d v,
$$

where $g^{\prime}$ is some new function of angular momentum. Assuming that $J$ is not a function of $r$ in the stellar halo, we drop all reference to $J$ from here on.

For a purely stellar system we have $E=\frac{1}{2} v^{2}+\Phi_{*}$, where $\Phi_{*}$ is the potential of the stars and is approximated by a Keplerian potential $\left(\Phi_{*} \propto-G M_{*} / r\right)$. Upon substitution into Equation (2) we recover the familiar result that $n(r) \propto r^{-3.5}$ in the halo of GCs. This result has been confirmed by direct $N$-body simulations (e.g., Baumgardt et al. 2002).

Our task here is simply to re-evaluate this integral with the addition of a DM potential, $\Phi_{\mathrm{DM}}$. The distribution function of weakly bound stars is unchanged with the addition of a dark halo since the derivation makes no reference to the form of the potential. We therefore have

$$
n(r) \propto r^{-2} \int_{E<0}\left|\frac{1}{2} v^{2}+\Phi_{*}+\Phi_{\mathrm{DM}}\right| d v,
$$

which upon integration becomes

$$
n(r) \propto r^{-2}\left(\Phi_{*}+\Phi_{\mathrm{DM}}\right)^{3 / 2}
$$

We assume a Navarro-Frenk-White (NFW) density profile for the dark halo that is motivated by collisionless $\Lambda$ CDM cosmological simulations (Navarro et al. 1996, 1997). The implied dark halo potential is

$$
\Phi_{\mathrm{DM}}=-G M_{\mathrm{DM}} g(c) \frac{\ln \left(1+r / r_{s}\right)}{r},
$$

where $M_{\mathrm{DM}}$ is the total dark halo "virial" mass, $c$ is the concentration defined as $c \equiv r_{v} / r_{s}$ where $r_{v}$ is the virial radius and $r_{s}$ is the scale radius, and $g(c)=[\ln (1+c)-c /(1+c)]^{-1}$. Over the physically relevant range of $2 \lesssim c \lesssim 10, g(c)$ varies from 2.3 to 0.7 .

Finally then, we have the following expression for the stellar density profile in the presence of a dark halo ${ }^{3}$ :

$$
n(r) \propto r^{-3.5}\left[1+\frac{M_{\mathrm{DM}}}{M_{*}} g(c) \ln \left(1+r / r_{s}\right)\right]^{3 / 2} .
$$

\footnotetext{
3 The contribution from unbound stars is not included here. We expect their contribution to be negligible because simulations consistently find that stars are unbound at a rate of $\sim 1 \%$ per relaxation time. Moreover, the density profile of the escapers is approximately $r^{-2}$ (Spitzer 1987), even in the presence of a dark halo, and so their presence would not impact our conclusions.
}



Figure 1. Stellar density profiles normalized to the density at $20 \mathrm{pc}$. Models are shown for several values of the dark halo-to-stellar mass ratio, $M_{\mathrm{DM}} / M_{*}$ (top panel), and dark halo scale radius, $r_{s}$ (bottom panel). In the top panel $r_{s}=250 \mathrm{pc}$, and in the bottom panel $M_{\mathrm{DM}} / M_{*}=10^{2}$. The blue and red dashed lines have logarithmic slopes of -3.5 and -2.0 , respectively.

(A color version of this figure is available in the online journal.)

For $M_{\mathrm{DM}} / M_{*} \ll 1$ we recover the familiar result of $n(r) \propto r^{-3.5}$. When the dark halo mass is significant, the profile can be decomposed into three regimes. At sufficiently small scales the first term in brackets in Equation (6) dominates over the second, and the profile scales as $r^{-3.5}$. At larger scales, the second term dominates, and it takes on two limits for $r$ smaller or larger than $r_{s}$. For $r<r_{s}$ the second term scales as $r$ and the total density profile then scales as $n(r) \propto r^{-2}$. At scales greater than $r_{s}$ the second term in brackets becomes shallow, and the resulting density profile consequently steepens.

In Figure 1 we show the expected stellar density profiles for several values of the parameters $M_{\mathrm{DM}} / M_{*}$ and $r_{s}$. For simplicity, we have fixed the virial radius to $r_{v}=1 \mathrm{kpc}$ although the models are insensitive to this simplification. Notice the strong sensitivity to $M_{\mathrm{DM}} / M_{*}$ and the weak sensitivity to the $r_{s}$ over the scales of interest. The weak sensitivity to $r_{s}$ is due to the fact that the logarithmic slope of the dark halo potential varies slowly across $r_{s}$.

Figure 1 demonstrates that the density profile over the range $10 \lesssim r \lesssim 100 \mathrm{pc}$ is very sensitive to the presence of a dark halo. Our derivation of the density profile is strictly appropriate only for the stellar halo of a GC, and so the profiles in Figure 1 will not represent real GCs on smaller scales. We have also ignored tidal stripping and the fact that the relaxation time at large scales may under certain circumstances be longer than the age of the universe. 
The derivation of the stellar density profile in the halo of GCs relies on the assumption that the two-body relaxation time is short compared to the age of the universe. We now verify under what conditions this assumption is valid. The radial dependence of the effective relaxation time in the stellar halo can be estimated as follows (see Lightman \& Shapiro 1978, for details). The relaxation time, $t_{r}$, scales as

$$
t_{r} \propto \frac{E^{2}}{D\left(\Delta E^{2}\right)}
$$

where $D$ is the diffusion rate and $E$ is the energy. The diffusion rate is simply the energy change per unit time, which, for stars in the halo, is (Binney \& Tremaine 1987)

$$
D\left(\Delta E^{2}\right) \propto \frac{\epsilon^{2}}{P},
$$

where $P$ is the orbital period and $\epsilon$ is the (small) change in energy per orbit. The key feature of stars in the halo is that they are on radial orbits that pass through the central regions of the GC. This fact implies that $\epsilon$ is approximately constant for stars in the halo; i.e., the change in energy per orbit does not depend on the apocentric distance of the orbit. Thus,

$$
t_{r} \propto E^{2} P
$$

Assuming that the potential is a combination of a Keplerian and an NFW dark halo, as we have throughout this section, we arrive at the following expression for the relaxation time:

$$
t_{r} \propto \frac{1}{\sqrt{r}}\left[1+\frac{M_{\mathrm{DM}}}{M_{*}} g(c)\left(\ln \left(1+r / r_{s}\right)-\frac{r / r_{s}}{1+r / r_{s}}\right)\right]^{3 / 2} .
$$

The relaxation time is a function of radius and DM-to-stellar mass ratio. These dependencies are illustrated in Figure 2. In this figure the relaxation time is scaled to the relaxation time at the half-mass radius. Notice first the counterintuitive result that in systems dominated by a Keplerian potential the relaxation time in the halo is actually a decreasing function of radius. As discussed in Lightman \& Shapiro (1978), this arises because the change in energy per orbit, $\epsilon$, is constant, while the energy of a star scales as $r^{-1}$. At greater clustocentric distances stars therefore require fewer orbits to change $E^{2}$ by of order itself. The increasing period with increasing distance is not sufficient to counteract this trend.

The addition of a dark halo modifies this behavior, such that larger DM contributions result in longer relaxation times. For sufficiently large DM fractions, the relaxation time will exceed the age of the universe. Figure 2 includes an upper bound provided by the age of the universe in units of the half-mass relaxation time of one GC we will consider in the next section, MGC1. For this cluster, the relaxation time in the halo does not exceed the age of the universe at $<6 R_{h}$, for $M_{\mathrm{DM}} / M_{*}=100$ and at $R<30 R_{h}$ for $M_{\mathrm{DM}} / M_{*}=10$. The very outer stellar halo of isolated GCs must be interpreted with these facts in mind.

Lightman \& Shapiro (1978) provided a simple derivation of the stellar density profile in the GC halo. In a steady state the net stellar flux through a spherical shell of radius $r$ must be constant, which implies that $n(r) r^{3} / t_{r}=$ const. This formula for $n(r)$, when combined with Equation (10), reproduces the stellar density profile derived earlier in this section (Equation (6)) in the limit where $E \sim 0$. This is not surprising because the principal assumption in both derivations is the existence of a steady state in the halo.

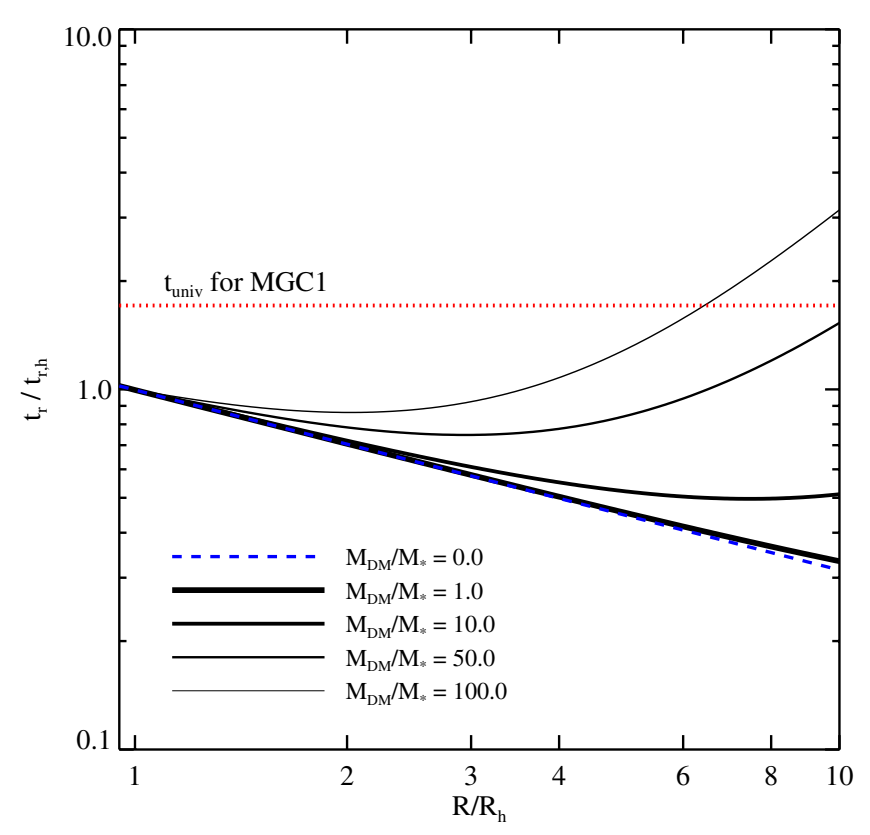

Figure 2. Relaxation time in units of the half-mass relaxation time, as a function of clustocentric distance. Results are shown for several values of the dark matterto-stellar mass ratio. The age of the universe is shown in units of the half-mass relaxation time of MGC1 (dotted line).

(A color version of this figure is available in the online journal.)

\subsection{Results}

As mentioned in the Introduction, most ancient GCs are on orbits that would likely have resulted in severe stripping of an extended dark halo, were they originally embedded in such halos. GCs at large galactocentric distance, in contrast, orbit within very weak tidal fields, and so one may expect these objects to have retained their dark halos, if they ever had them.

Two GCs are particularly noteworthy in this regard: NGC 2419 in the MW and MGC1 in M31. NGC 2419 resides at $90 \mathrm{kpc}$ from the center of our Galaxy, has a half-mass radius and tidal radius of $20 \mathrm{pc}$ and $230 \mathrm{pc}$, respectively, and a $V$-band luminosity of $5 \times 10^{5} L_{\odot}$ (Harris 1996), which implies a total stellar mass of $\approx 10^{6} M_{\odot}$. Bellazzini (2007) recently measured the stellar surface density of NGC 2419 to $200 \mathrm{pc}$. The core and half-mass relaxation times of this GC are 9 and 35 Gyr, respectively.

Mackey et al. (2010) recently measured structural and photometric properties of MGC1, from which we have learned the following. MGC1 resides at approximately $200 \mathrm{kpc}$ from M31, and is therefore the most isolated GC known in the Local Group. It has a $V$-band luminosity of $4 \times 10^{5} L_{\odot}$ and thus a stellar mass of $\approx 10^{6} M_{\odot}$, a half-mass radius of $\approx 7.5 \mathrm{pc}$, and an indeterminate tidal radius. Mackey et al. have measured the stellar surface density for MGC1 out to an impressive 900 pc. We can estimate the core and half-mass relaxation times of MGC1 by scaling the relaxation times of NGC 2419 by the $3 / 2$ power of the ratio of their half-mass radii. Doing so yields core and half-mass relaxation times of 2 and $8 \mathrm{Gyr}$, respectively.

In Figure 3, we compare the observed stellar surface density profiles of NGC 2419 and MGC1 to our model density profile for several values of the dark halo-to-stellar mass ratio, $M_{\mathrm{DM}} / M_{*}$. We have fixed $r_{s}=250 \mathrm{pc}$ and hence $c=4$ for simplicity. Such a low value of $c$ is expected for low-mass halos that formed at high redshift (Navarro et al. 1997). Data are only shown for $R_{p}>R_{h}$. On scales smaller than roughly the half-mass radius 


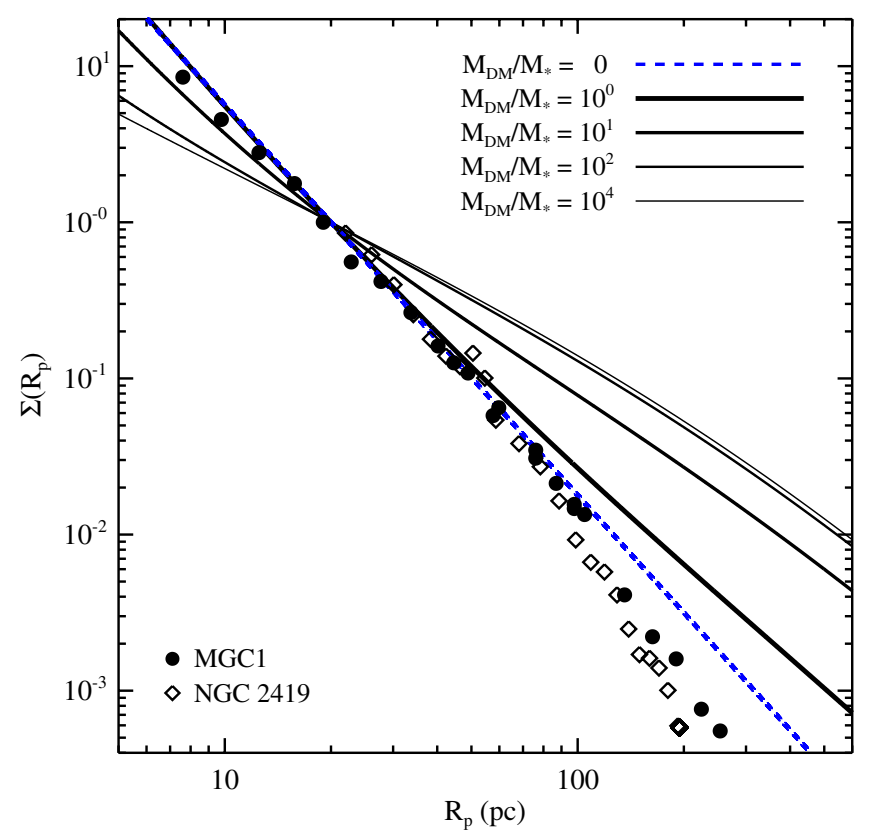

Figure 3. Stellar surface density profiles normalized to the surface density at 20 pc. Our models, which include a stellar component of mass $M_{*}$ embedded within a dark halo of mass $M_{\mathrm{DM}}$, are shown as lines for a range of mass ratios. These models are compared to data from the GC MGC1 located in the outer halo of M31 (Mackey et al. 2010) and the GC NGC 2419 located in the outer halo of the MW (Bellazzini 2007). Data are only plotted for $R_{p}>R_{h}$. The blue dashed line has a logarithmic slope of -2.5 and is the predicted surface density profile for a pure stellar system.

(A color version of this figure is available in the online journal.)

our assumptions break down (as demonstrated by direct $N$-body simulations; Baumgardt et al. 2002).

Over the range $10 \mathrm{pc} \lesssim R_{p} \lesssim 100 \mathrm{pc}$ the data are consistent with the predictions for a pure stellar system; models with a massive extended dark halo are strongly disfavored. On larger scales deviation between the data and models is apparent, which may be due to tidal stripping or the ongoing assembly of the outer stellar halo.

Our model assumes that a steady state has been achieved in the stellar halo via two-body relaxation effects. MGC1 has a half-mass relaxation time shorter than the age of the universe, and so our technique can be readily applied to this system. Indeed, in the absence of a dark halo the effective relaxation time decreases with radius (Figure 2) and so we expect a welldeveloped stellar halo around MGC1. For models with massive dark halos (i.e., large $M_{\mathrm{DM}} / M_{*}$ ), our results strictly apply only to the inner several half-mass radii-at larger radii the effective relaxation time becomes longer than the age of the universe. However, even within $1<R / R_{h}<2$ the data strongly favor models with minimal DM halos. Moreover, the data at larger radii are naturally explained by assuming that the stellar halo is fully populated by relaxation effects in the absence of an embedded dark halo.

Conclusions regarding NGC 2419 must be made with greater caution than MGC1, since NGC 2419 has a present $t_{r}$ that is longer than the age of the universe. In this case it is less clear that our model should apply at all. We are left only with the striking agreement between the density profile of the halo of this GC and the model prediction that includes no dark halo. This strongly suggests, but does not rigorously demonstrate, that NGC 2419 is not surrounded by a massive dark halo.

Our results are consistent with Baumgardt et al. (2009), who concluded that if a dark halo surrounds NGC 2419, it cannot be more massive than $10^{7} M_{\odot}$ (this is equivalent to a limit of $M_{\mathrm{DM}} / M_{*}<10$ for this GC). These latter results were based on the measured velocity dispersion profile of NGC 2419 over the range $10 \mathrm{pc} \lesssim R_{p} \lesssim 60 \mathrm{pc}$.

\section{DISCUSSION}

In the previous section we argued that the observed stellar surface density profiles of the GCs NGC 2419 and MGC1 place strong constraints on the existence of extended dark halos surrounding these GCs. The data are consistent with no dark halo, and a firm upper limit on the dark halo-mass-to-stellar mass ratio is $M_{\mathrm{DM}} / M_{*}<1$. The conclusions are strongest for MGC1 because it, unlike NGC 2419, has a core relaxation time much less than the age of the universe.

This upper limit effectively rules out the possibility that these GCs formed at the center of their own dark halos, under the assumption that these GCs have evolved in weak tidal fields throughout their lifetimes. This assertion is based on the following argument. If these GCs did form within their own dark halos and subsequently experienced little tidal stripping, then the smallest possible value for $M_{\mathrm{DM}} / M_{*}$ would be $\left(1-f_{b}\right) / f_{b}$ where $f_{b}$ is the universal baryon fraction. Constraints from the cosmic microwave background imply $f_{b}=0.17$ (Komatsu et al. 2009), and so $M_{\mathrm{DM}} / M_{*}>5$. Of course, less than $100 \%$ star formation efficiency, which is expected, would only increase this lower limit. Our upper limit of $M_{\mathrm{DM}} / M_{*}<1$ therefore strongly suggests that these GCs did not form within their own dark halos.

Observations of the outer stellar profile of isolated GCs are very sensitive to a dark halo because a dark halo, were it to exist, should have a half-mass radius much larger than the GC stellar half-mass radius. This fact also explains why it has historically been so difficult to obtain strong constraints on the presence of a dark halo with kinematic data, even with data extending to several tens of parsecs (e.g., Lane et al. 2010). An NFW DM halo with a virial mass of $10^{8} M_{\odot}$ has a mass of only $10^{6} M_{\odot}$ within $50 \mathrm{pc}$, assuming $c=2$ (or within $10 \mathrm{pc}$ assuming $c=20$ ). For NGC 2419, which has a stellar mass of $\approx 10^{6} M_{\odot}$, the presence of such a halo would be very difficult to distinguish from the uncertain corrections required to account for low-mass stars and stellar remnants, based on data that only extend to several tens of parsecs.

In recent years it has become clear that most, if not all, GCs harbor internal spreads in the abundance of light elements, including $\mathrm{CNO}, \mathrm{Na}, \mathrm{Mg}$, and $\mathrm{Al}$ (see Gratton et al. 2004, for a review). Several authors have appealed to GC formation at the center of extended dark halos to account for these puzzling observations (e.g., Freeman 1993; Bekki \& Norris 2006; Bekki et al. 2007; Böker 2008; Carretta et al. 2010a). One of the advantages of forming GCs at the center of massive dark halos is that they are much less susceptible to ram pressure stripping, and, the argument goes, are therefore better able to retain the gaseous material necessary to account for the observed internal abundance spreads. As discussed in Conroy \& Spergel (2011), this line of reasoning is likely incorrect because the formation environments of the ancient GCs differed substantially from their present-day environment. The results in this work provide strong independent confirmation that indeed GCs which harbor multiple stellar populations do not (or need not) form within extended dark halos.

While the current evidence disfavors typical GCs from having formed at the center of their own dark halos, there is some reason 
to suspect that perhaps some of the most massive GCs did indeed form in this way. M54 is the most striking example, as it resides at the center of the disrupting Sagittarius galaxy, and will in the future likely orbit freely through the Galaxy (although recent evidence suggests that M54 resides at the center of Sagittarius because of dynamical friction, not because it formed there; see Bellazzini et al. 2008 for details). Other candidates for this formation mechanism include $\omega$ Cen, M22, NGC 1851, and G1 in M31, all of which show internal spreads in the Fe-peak elements. These GCs must have formed in deep potential wells in order to retain the Fe generated from Type Ia supernovae $(\mathrm{SNe})$. Nuclear star clusters may be the precursors of these massive GCs. The most massive GCs in external galaxies also appear to be self-enriched in Fe (Strader \& Smith 2008; Bailin \& Harris 2009), although the fact that their photometric properties join seamlessly with the less massive clusters suggests that GCs of all masses share a common origin unrelated to dark halos. Detailed simulations will be necessary to conclude whether or not GCs can self-enrich in $\mathrm{SNe}$ products without surrounding dark halos.

Olszewski et al. (2009) recently reported the discovery of a 500 pc stellar halo surrounding the GC NGC 1851. Over the projected radial range of $50-250 \mathrm{pc}$, these authors find a projected stellar density profile of $\Sigma \propto r^{-1.24 \pm 0.66}$. This measured profile agrees remarkably well with models that include a massive dark halo $\left(M_{\mathrm{DM}} / M_{*}>10^{2}\right)$, which predict a logarithmic slope of -1.4 over the same radial range. NGC 1851 currently resides only $17 \mathrm{kpc}$ from the Galactic center and, according to Olszewski et al. (2009), has a period of $0.4 \mathrm{Gyr}$ and a perigalacticon of only $5 \mathrm{kpc}$. The interpretation of the density profile of weakly bound stars in this cluster is therefore greatly complicated by the stronger tidal fields it experiences and the effect of disk shocking as it crosses the MW disk five times per Gyr. The lack of any tidal tails is also peculiar given its orbit. As noted above, NGC 1851 shows evidence for an internal spread in Fe abundance (Carretta et al. 2010b), and so is a potential candidate for being the remnant of a disrupted dwarf galaxy. Future work on the orbit and stellar population of this cluster may reveal important clues regarding its formation. Radial velocity measurements would be especially valuable, as they should be able to distinguish between a stellar halo formed from tidal effects and one formed from loosely bound stars on radial orbits.

Recently, Cohen et al. (2010) measured iron and calcium abundances of stars in NGC 2419. These authors report the discovery of an internal spread in $\mathrm{Ca}$ abundances in this cluster, but no spread in Fe. If confirmed, this result suggests that NGC 2419 was able to retain Type II SNe ejecta, which is difficult to understand unless this cluster was once embedded within a much deeper potential well than it is currently. It could of course be the case that the stars in NGC 2419 simply formed from a chemically heterogenous molecular cloud, or that the cluster contained many more stars at birth. As with NGC 1851, future work on the abundance variations of the stars within NGC 2419 and a detailed analysis of its orbit will provide essential clues into the origin of this puzzling GC.

We conclude by recalling a central assumption in the present work: that outer halo GCs have evolved in isolation throughout their lifetimes. Unless these GCs formed in intergalactic space, they likely once resided within larger protogalactic fragments that have since been tidally destroyed. We can say little with confidence regarding the influence of the birth environment on the structure of these GCs. Mass lost from these young
GCs during their first $\sim 1$ Gyr of evolution would result in an expansion of the system due to the loss of binding energy (Kroupa \& Boily 2002; Marks \& Kroupa 2010). These effects complicate the interpretation of the outer stellar halo of the GCs NGC 2419 and MGC1. Nonetheless, the tenuous nature of their stellar halos and the observed similarity in their radial profiles strongly suggests that they are being continuously populated by two-body relaxation effects.

We thank Dougal Mackey for providing his data on MGC1, Jay Strader for fruitful conversations, and Dougal Mackey and Scott Tremaine for comments on an earlier draft. The referee is thanked for insightful comments that improved the quality of the manuscript. This work made extensive use of the NASA Astrophysics Data System and of the astro-ph preprint archive at arXiv.org, and was supported in part by NSF grants AST0907890 and AST-0707731 and NASA grants NNX08AK43G and NNA09DB30A.

\section{REFERENCES}

Ashman, K. M., \& Zepf, S. E. 1992, ApJ, 384, 50

Bailin, J., \& Harris, W. E. 2009, ApJ, 695, 1082

Baumgardt, H., Côté, P., Hilker, M., et al. 2009, MNRAS, 396, 2051

Baumgardt, H., Hut, P., \& Heggie, D. C. 2002, MNRAS, 336, 1069

Bekki, K., Campbell, S. W., Lattanzio, J. C., \& Norris, J. E. 2007, MNRAS, 377,335

Bekki, K., \& Norris, J. E. 2006, ApJ, 637, L109

Bellazzini, M. 2007, A\&A, 473, 171

Bellazzini, M., Ibata, R. A., Chapman, S. C., et al. 2008, AJ, 136, 1147

Binney, J., \& Tremaine, S. 1987, Galactic Dynamics (Princeton, NJ: Princeton Univ. Press), 747

Böker, T. 2008, ApJ, 672, L111

Böker, T., Sarzi, M., McLaughlin, D. E., et al. 2004, AJ, 127, 105

Bromm, V., \& Clarke, C. J. 2002, ApJ, 566, L1

Carretta, E., Bragaglia, A., Gratton, R. G., et al. 2010a, A\&A, 516, A55

Carretta, E., Gratton, R. G., Lucatello, S., et al. 2010b, ApJ, 722, L1

Cohen, J. G., Kirby, E. N., Simon, J. D., \& Geha, M. 2010, ApJ, 725, 288

Conroy, C., \& Spergel, D. N. 2011, ApJ, 726, 36

Fall, S. M., \& Rees, M. J. 1985, ApJ, 298, 18

Freeman, K. C. 1993, in ASP Conf. Ser. 48, The Globular Cluster-Galaxy Connection, ed. G. H. Smith \& J. P. Brodie (San Francisco, CA: ASP), 608 Gratton, R., Sneden, C., \& Carretta, E. 2004, ARA\&A, 42, 385

Grillmair, C. J., Freeman, K. C., Irwin, M., \& Quinn, P. J. 1995, AJ, 109, 2553

Gunn, J. E. 1980, in Globular Clusters, ed. D. Hanes \& B. Madore (Cambridge: Cambridge Univ. Press), 301

Harris, W. E. 1996, AJ, 112, 1487

Harris, W. E., \& Pudritz, R. E. 1994, ApJ, 429, 177

Holtzman, J. A., Faber, S. M., Shaya, E. J., et al. 1992, AJ, 103, 691

Komatsu, E., Dunkley, J., Nolta, M. R., et al. 2009, ApJS, 180, 330

Kravtsov, A. V., \& Gnedin, O. Y. 2005, ApJ, 623, 650

Kroupa, P., \& Boily, C. M. 2002, MNRAS, 336, 1188

Lane, R. R., Kiss, L. L., Lewis, G. F., et al. 2010, MNRAS, 406, 2732

Lightman, A. P., \& Shapiro, S. L. 1978, Rev. Mod. Phys., 50, 437

Mackey, A. D., Ferguson, A. M. N., Irwin, M. J., et al. 2010, MNRAS, 401, 533

Marks, M., \& Kroupa, P. 2010, MNRAS, 406, 2000

Mashchenko, S., \& Sills, A. 2005, ApJ, 619, 258

Moore, B. 1996, ApJ, 461, L13

Muratov, A. L., \& Gnedin, O. Y. 2010, ApJ, 718, 1266

Navarro, J. F., Frenk, C. S., \& White, S. D. M. 1996, ApJ, 462, 563

Navarro, J. F., Frenk, C. S., \& White, S. D. M. 1997, ApJ, 490, 493

Odenkirchen, M., Grebel, E. K., Dehnen, W., et al. 2003, AJ, 126, 2385

Olszewski, E. W., Saha, A., Knezek, P., et al. 2009, AJ, 138, 1570

Peebles, P. J. E. 1984, ApJ, 277, 470

Spitzer, L. 1987, in Dynamical Evolution of Globular Clusters, ed. L. Spitzer (Princeton, NJ: Princeton Univ. Press), 191

Spitzer, L., Jr., \& Hart, M. H. 1971, ApJ, 164, 399

Spitzer, L., Jr., \& Shapiro, S. L. 1972, ApJ, 173, 529

Strader, J., \& Smith, G. H. 2008, AJ, 136, 1828

Walcher, C. J., Böker, T., Charlot, S., et al. 2006, ApJ, 649, 692

Walcher, C. J., van der Marel, R. P., McLaughlin, D., et al. 2005, ApJ, 618, 237

Whitmore, B. C., \& Schweizer, F. 1995, AJ, 109, 960

Whitmore, B. C., Zhang, Q., Leitherer, C., et al. 1999, AJ, 118, 1551 